This item was submitted to Loughborough's Research Repository by the author.

Items in Figshare are protected by copyright, with all rights reserved, unless otherwise indicated.

\title{
Optimising the quality of an SfM-MVS slope monitoring system using fixed cameras
}

\section{PLEASE CITE THE PUBLISHED VERSION}

https://doi.org/10.1111/phor.12288

\section{PUBLISHER}

Wiley

\section{VERSION}

AM (Accepted Manuscript)

\section{PUBLISHER STATEMENT}

This is the peer reviewed version of the following article: Parente, L., Chandler, J.H. and Dixon, N. (2019), Optimising the quality of an SfM-MVS slope monitoring system using fixed cameras. The Photogrammetric Record, 34 (168), pp. 408-427 which has been published in final form at https://doi.org/10.1111/phor.12288. This article may be used for non-commercial purposes in accordance with Wiley Terms and Conditions for Use of Self-Archived Versions.

\section{LICENCE}

CC BY-NC-ND 4.0

\section{REPOSITORY RECORD}

Parente, Luigi, Jim Chandler, and Neil Dixon. 2019. "Optimising the Quality of an Sfm-mvs Slope Monitoring System Using Fixed Cameras”. figshare. https://hdl.handle.net/2134/9816734.v1. 


\title{
OPTIMISING THE QUALITY OF AN SFM-MVS SLOPE MONITORING SYSTEM USING FIXED CAMERAS
}

\author{
LUIGI PARENTE* (1.parente@lboro.ac.uk) \\ JIM H. CHANDLER (j.h.chandler@lboro.ac.uk) \\ NEIL DiXON (N.Dixon@lboro.ac.uk) \\ School of Architecture, Building and Civil Engineering, Loughborough University, UK \\ * Corresponding author
}

\section{Abstract}

The quality of $3 D$ scene reconstruction and monitoring through structurefrom-motion multi-view stereo (SfM-MVS) depends on critical key factors, including camera calibration and image network geometry. The goal of this paper is to examine the monitoring ability of an SfM-MVS workflow based on four or more ground-based digital single-lens reflex (DSLR) cameras and to estimate differences when adopting both fixed and variable camera positions and orientations. This was achieved by conducting work on a scaled laboratory test field and a sea cliff. Tests demonstrate that a monitoring system using just four fixed cameras can achieve valuable monitoring capabilities and tolerate imperfections in the camera calibration. Furthermore, such a configuration can achieve accuracies comparable to terrestrial laser scanning (TLS) and dronebased photogrammetry. The study demonstrates that minimising registration errors between point clouds is critical. The 'registration SIFT' approach could resolve such problems.

KEYWORDS: calibration, change detection, monitoring, network geometry, photogrammetry, structure from motion (SfM)

\section{INTRODUCTION}

ACCURATE CAMERA CALIBRATION, an appropriate image-acquisition strategy and precise model registration remain critical stages in a photogrammetric workflow to extract high-accuracy measurements. Developing a photogrammetric system for monitoring slope instability based on a fixed set of digital single-lens reflex (DSLR) cameras provided the opportunity to re-examine these stages. The term 'fixed' is adopted in this paper to describe a photogrammetric setup which remains stationary throughout the monitoring period. Previous work involving a structure-from-motion photogrammetric workflow and camera calibration is reviewed before describing the methodology developed to test the monitoring 
of the proposed system based upon four fixed cameras. Specifically, the purpose of this study is to investigate the performance of a photogrammetric monitoring system when a fixed camera configuration is used during image acquisition, even with poorly calibrated DSLR cameras. The quality of the monitoring system when processing a variable number of image locations $(4-10)$ is assessed as well. Finally, a potentially useful approach to eradicate errors due to poor model registration is described.

\section{LITERATURE REVIEW}

Recent technological advances have contributed to the development of inexpensive and fast 4D data acquisition and processing approaches. For instance, the structure-frommotion (SfM) workflow (Snavely et al., 2006) is a photogrammetric technique (Ullman, 1979) which, in combination with multi-view stereo (MVS) algorithms (Seitz et al., 2001), produces a dense 3D point cloud using images acquired with non-metric cameras. This technique, described as SfM-MVS, provides a valuable option for a range of low-budget monitoring applications. The availability of a wide range of inexpensive digital single-lens reflex (DSLR) cameras, together with commercial and non-commercial software to automatically capture three-dimensional structures, support the increasing use of this technique. In a recent review presented by Eltner et al. (2016), it was found that 65 scientific works adopted SfM for a range of geoscientific applications between 2012 and 2015. Monitoring change by comparing dense 3D point clouds obtained by implementing SfMMVS produced results comparable to more established techniques, including traditional field survey methods (Gillan et al., 2017), conventional photogrammetry (Bakker and Lane, 2017) and terrestrial laser scanner (TLS) (Nouwakpo et al., 2016). Despite this considerable potential, some critical factors can easily affect the quality of the 3D scene generated with an SfM-MVS workflow, introducing constraints and limitations.

General advice for efficient image acquisition methods is presented in Carrivick et al. (2016), based on a range of SfM-MVS applications. This review demonstrates that depending on the site (occlusion, shape complexity and scale), the desired detail and the time users are willing to wait, tens to hundreds of pictures are typically acquired. The review by Eltner et al. (2016) showed that in 38 studies implementing the SfM-MVS workflow, only two used fewer than 10 images. Also, it was noted that by adopting larger baselines between images, glancing ray intersections are avoided, resulting in an increase in accuracy. Piermattei et al. (2015) discussed that a better quality of SfM-MVS output is achieved by adopting a higher number of images and decreasing the baseline as this produces less change in feature appearance, thus facilitating implementation of the matching algorithm. Therefore, a trade-off between feature appearance change and glancing rays must be considered when choosing a baseline. Micheletti et al. (2015) demonstrated that increasing the number of multi-scale images produces denser point clouds, improving the model accuracy. The latter study also stated that 'smaller image sets can still provide acceptable accuracies, provided their spatial distribution is adequate'. The typical acquisition geometry used in previous work is a multistation configuration where the same camera captures a single shot at each station. Recent studies investigated the use of stereo cameras in fixed positions for monitoring purposes (James and Robson, 2014; Roncella et al., 2014). A multistation approach with fixed cameras can produce accurate results using both DSLR and trail cameras (Eltner et al., 2017; Mallalieu et al., 2017). 
In order to extract precise and reliable 3D metric information from images captured with non-metric cameras, accurate interior orientation parameters must be estimated. In fact, uncertainty in the parameters describing the internal geometry of cameras not designed for metric purposes (for example consumer-grade digital cameras) can compromise the photogrammetric object restitution (Fryer et al., 2007). Camera calibration methods have evolved over time (Mason et al., 1997; Heikkilä, 2000; Remondino and Fraser, 2006; Douskos et al., 2007). The most common calibration procedure used with consumer-grade digital cameras is self-calibration via the bundle adjustment (Brown 1971; Kenefick et al. 1972). Such procedures use images captured specifically for the task, typically of a laboratory testfield. Most SfM workflows integrate the self-calibration of cameras, providing the advantages of automation and ease of use. It has to be noted that whilst some software use simplified calibration models (for example, only one distortion parameter is estimated), 'proper' photogrammetric software can model the inner geometry of a camera with a range of parameters including the principal distance, the principal-point offset and corrections for radial $\left(k_{1}, k_{2}, k_{3}\right)$ and decentring distortion $\left(p_{1}, p_{2}\right)$ (Kenefick et al., 1972). Unfortunately, these estimates may be inaccurate; Gruen and Beyer (2001) indicate the radial distortion is the most critical of all interior parameters. Disregarding radial distortion or using inaccurate radial distortions parameter (for example a $20 \%$ variation of $k_{1}$ ) can significantly decrease the accuracy of any photogrammetric project (Wackrow and Chandler, 2008).

Internal camera geometry can vary over time and frequent calibration of cameras is advisable for high object-space reconstruction accuracies (Habib et al., 2014). Major reasons for variation include mechanical or thermal change (Gülch, 1984; Habib and Morgan, 2005), logistics (transportation and repeated power cycling) and mode of operation (lenses are changed or refocused) (Sanz-Ablanedo et al., 2010). Two approaches are used to cope with geometric instability, parameterisation and camera stabilisation (Rieke-Zapp et al., 2009).

This review encourages investigation into the use of a SfM-MVS workflow with fixed multiple cameras and a lower number of images for geomorphic monitoring. Two hypotheses were tested during this work and are described in this paper: (1) the influence of a poorly calibrated camera model is negated when the camera configuration is identical over the whole monitoring period; and (2) an SfM-MVS workflow can produce dense and precise point clouds, even when a low number of images is used. A laboratory experiment was designed to investigate the above assumptions and was supported by a full-scale field study. Furthermore, the need to find a robust approach to cope with model registration is discussed.

\section{TEST Sites AND METHOdOLOGY}

Two series of tests were conducted, one in a controlled laboratory environment and the other in the field. These allowed the monitoring abilities of a consumer-grade digital camera system, based upon four DSLR cameras, to be evaluated. The laboratory environment enabled the impact of fixed and varied acquisition networks, with both precise and inaccurately determined internal camera geometry, to be investigated. The fieldwork permitted the system to be tested on a real site at a larger scale and when a variable number of images are captured. 


\section{Experimental Environment}

For the laboratory experiment, a scaled model was designed and built at the Sir Frank Gibb Laboratories at Loughborough University, UK (Fig. 1). The model attempted to replicate a steep vegetation-free slope, with the following dimensions: $1.5 \mathrm{~m}$ in length, $0.6 \mathrm{~m}$ in height and $0.5 \mathrm{~m}$ in width, with a gradient of $60^{\circ}$. It was mainly composed of poorly sorted sandy gravel, low in fine sharp sand and with a few pebbles added to the surface. The model geometry and material used were chosen for two reasons: (1) they provided ideal conditions for SfM-MVS algorithms to generate a dense point cloud; and (2) it was easy to introduce localised displacement for testing the monitoring ability of the system. Nine photogrammetric PhotoModeler RAD (ringed automatically detected or coded targets) were distributed around the area of interest (Fig. 1) and provided ground control points (GCPs). A robotic total station was employed to determine their coordinates in a local rectangular Cartesian coordinate system. Observations were determined from two stations and a least squares "variation of coordinates" program (StarNet V7) was used to estimate coordinates with a precision of $\pm 1 \mathrm{~mm}(1 \sigma)$. Four Nikon D80 cameras equipped with $24 \mathrm{~mm}$ lenses, tripods and remote exposure-triggering devices were used to capture sharp and unblurred images. The Nikon D80 is a low-price 10 megapixel consumer-grade DSLR camera (sensor with 3872 x 2592 pixels). All cameras were set on 'aperture priority' mode and identical settings ensured (ISO, exposure bias, and so on). Tripods were positioned $2 \mathrm{~m}$ from the laboratory model, with a camera baseline of $2.2 \mathrm{~m}$ whilst ensuring that the camera axes intersected the object plane at the same point (Fig. 1). This camera configuration provided an efficient imaging geometry (Wackrow and Chandler, 2008) avoiding an overemphasised convergence which may affect the quality of the image matching (Eltner et al., 2016).

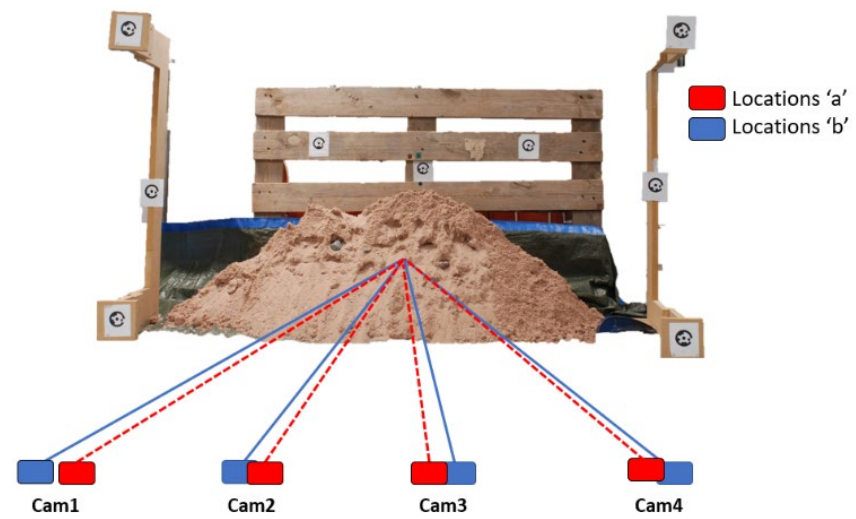

FIG. 1. Four cameras captured images of the laboratory model with two slightly different network geometries (locations: 'a' (red) are fixed; locations 'b' (blue) are variable). The nine PhotoModeler coded targets can be seen on the surrounding structure.

Photographs of the test field were acquired at three epochs with the four cameras positioned in two locations before and after introducing change on the slope. In this way a total of four 3D point clouds were generated and compared to evaluate differences (Table I). In order to clarify the tests conducted, the two cameras locations adopted are indicated with letters 'a' and ' $b$ '. Specifically, ' $a$ ' corresponds to the initial position of the four 
cameras, whilst ' $b$ ' indicates that the four cameras are in a new position, slightly different in both location and orientation than position ' $a$ '. For the second camera positions (b) a small displacement of 3 to $6 \mathrm{~cm}$ from the initial position ' $\mathrm{a}$ ' was applied to the cameras. Images for epoch1 and epoch2 captured the same slope as the surface was not altered. Epoch3 was captured after small slope displacements were manually introduced in three small localised areas. With this approach, the quality of monitoring was evaluated in the following scenarios (Table I):

(1) stable slope and fixed cameras (epoch1a - epoch2a);

(2) stable slope and variable cameras (epoch $2 a-$ epoch $2 b)$;

(3) displacement on the slope and fixed cameras (epoch2b-epoch $3 b$ ); and

(4) displacement on the slope and variable cameras (epoch2a - epoch3b).

TABLE I. Epochs compared in the laboratory experiment test to evaluate sensitivity of the system to fixed (a) or variable (b) camera set-ups.

\begin{tabular}{cccc}
\hline Epoch & $\begin{array}{c}\text { Camera } \\
\text { set-up }\end{array}$ & $\begin{array}{c}\text { Slope } \\
\text { condition }\end{array}$ & Comparison \\
\hline 1 & $\mathrm{a}$ & Stable & $1 \mathrm{a}-2 \mathrm{a}$ \\
2 & $\mathrm{a}$ & Stable & $2 \mathrm{a}-2 \mathrm{~b}$ \\
2 & $\mathrm{~b}$ & Stable & $2 \mathrm{~b}-3 \mathrm{~b}$ \\
3 & $\mathrm{~b}$ & Displacement & $2 \mathrm{a}-3 \mathrm{~b}$ \\
\hline
\end{tabular}

\section{Fieldwork}

Fieldwork was carried out to prove that the minimum four-image configuration could be effective at full scale, whilst providing the opportunity to consider the impact of including up to ten images. This was investigated on a relatively small section (approximately 25 x 20 metres) of the Spittles cliffs, situated in Lyme Regis, England (Fig. 2 ). This cliff was chosen as it represents a typical user scenario under non-controlled conditions, providing the opportunity to detect small geomorphic change (for example rockfall and hydraulic action at the base of the cliff) over a short temporal baseline. This cliff section, mainly composed of Greensand that rests on Lower Lias clays with interbedded limestone layers (May, 2003), is situated along an actively eroding coastline. Extensive monitoring of ground movement has been undertaken since the mid 1990s (Fort et al., 2007). Monitoring strategies adopted include pointwise surveying techniques, involving Global Navigation Satellite System (GNSS) and total station monitoring ground markers. These techniques do not provide a useful benchmark to assess the quality of the proposed SfM approach, due to inconsistent spatial resolution and temporal data acquisition rate. A combination of complementary techniques, including high-resolution terrestrial laser scanning (TLS), unmanned aerial vehicle (UAV) photogrammetry and a robotic total station, were employed to facilitate $3 \mathrm{D}$ model alignment and comparison with more established techniques. 


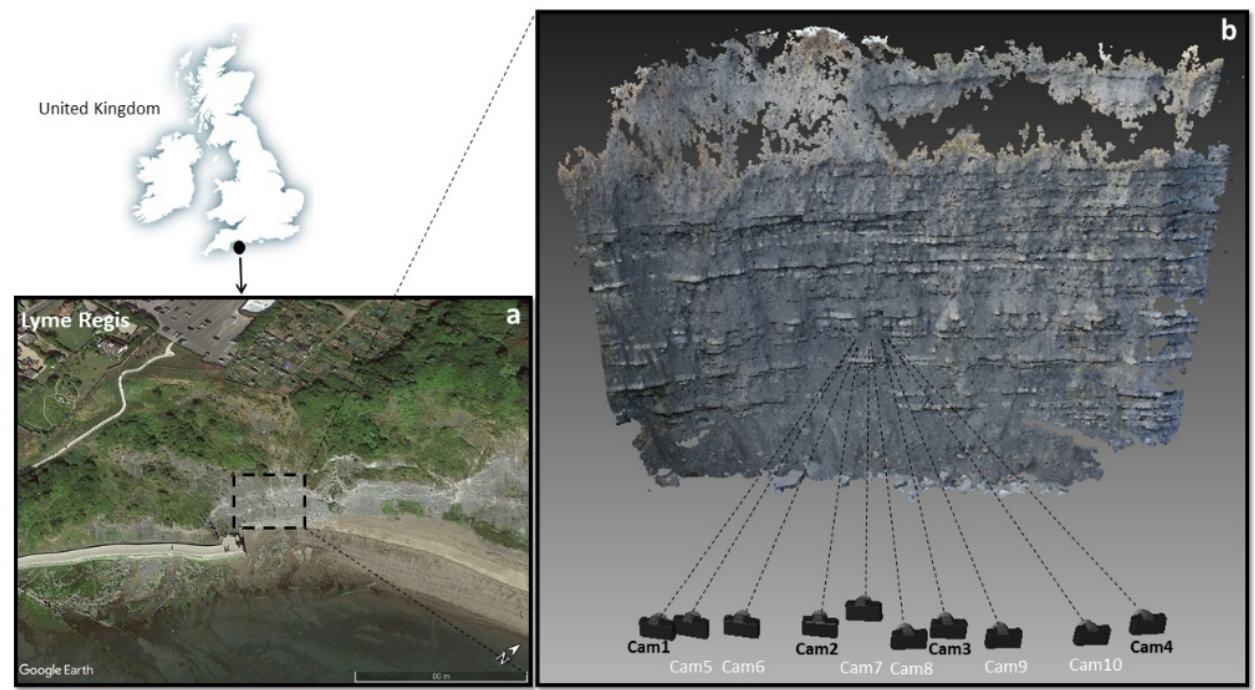

FIG. 2. Overview of the Lyme Regis (England) test site. (a) The black dashed rectangle shows position of the investigated coastal cliff. (b) Camera network geometry adopted to capture images of the site.

Image acquisition was conducted using a Nikon D80 DSLR equipped with a Nikkor lens with a fixed focal length of $24 \mathrm{~mm}$ and mounted obliquely on standard camera tripods. A remote exposure triggering control was also used to acquire sharp imagery, free of blur. Ensuring a constant distance of about $30 \mathrm{~m}$ between camera and cliff, a total of four images were captured by moving the camera parallel to the cliff face. A mildly convergent image configuration was employed (Cam1 to Cam4 in Fig. 2(b)) and a camera baseline of approximately $30 \mathrm{~m}$ was used. This simple approach was repeated on the following dates: 22nd and 23rd August 2017 (epoch1 and epoch2) and 17th February 2018 (epoch3). Epoch1 was captured after positioning 10 photogrammetric targets across the site, the position of the targets being measured using a reflectorless total station. On the first day (22nd August 2017) two additional data capture techniques were adopted. A TLS (Leica ScanStation 2) was used to capture a dense point cloud of the photographed site with an approximate resolution of $5 \mathrm{~mm}$. The laser measurement was carried out from a single location situated about $30 \mathrm{~m}$ from the cliff face. A UAV photogrammetric survey was also conducted, capturing 29 images using a DJI Phantom 3 Professional equipped with a 12 megapixels digital camera. Epoch2 data was collected the following day after the removal of targets. A targetless approach was also adopted on 17th February 2018, capturing epoch3 data. Installing stable targets for a long period was considered challenging due to strong winds, sea tides and storms, in addition to the geological characteristics of the slope. For all epochs, the following efforts were made to ensure the same camera positions were maintained following a consistent acquisition procedure:

(1) setting the tripods on easily identifiable locations based on stable features on site (such as the adjacent seawall and bare rock platform);

(2) ensuring the camera height was set to $1.7 \mathrm{~m}$ by manual measurement from the ground to the tripod plate; and

(3) securing the exterior camera orientation by keeping a convergent configuration by pointing the camera to the same stable feature on the cliff. 
It was not possible to install fixed tripods on the site and the camera locations over different epochs may differ by approximately 0.5 to $1 \mathrm{~m}$. The third epoch's dataset was collected to investigate the sensitivity of the approach to the number of images processed. For this reason, besides capturing images from the four locations (Cam 1 to Cam4), with epoch3 an additional six images were captured from additional locations (Cam5 to Cam10) as shown in Fig. 2(b).

\section{Data Processing, Registration and Comparison}

For both test sites (laboratory and field test), the methodology to determine 3D displacements and measurement accuracy involved the following stages:

(1) run the SfM-MVS workflow to generate dense point clouds corresponding to subsequent observation epochs;

(2) employ a cloud alignment procedure;

(3) cloud cropping; and

(4) estimate distances between closest points.

The SfM-MVS photogrammetric processing was implemented entirely in PhotoModeler, a commercial 3D scene reconstruction software which has already proved its efficiency for geoscientific applications (Irvine-Fynn et al., 2014). For each set of images, a five-step procedure was used to generate a dense point cloud:

(1) importing images;

(2) specifying the type of camera(s) used and calibration parameters;

(3) estimating camera positions using the bundle adjustment algorithm;

(4) increasing the point cloud density (MVS process); and

(5) scaling and orientating the model.

While steps (3) and (4) (Table III) remain unchanged for the 3D reconstructions of both sites, some differences apply, as outlined in Table II. In step (2), for the purpose of the present study, two camera calibration options were considered. The first included a 'full calibration' procedure which allowed the determination of the intrinsic parameters to a high degree of accuracy through a self-calibration via the bundle adjustment method (Kenefick et al., 1972). This procedure involved the collection of a set of images of a planar testfield consisting of more than 200 PhotoModeler RAD targets. The camera lens was initially focused and locked using adhesive tape. This stabilisation measure was maintained for all photograph acquisition at both test sites. However, it must be noted that the lens focus ring was locked at different positions for the laboratory and field sites $(2 \mathrm{~m}$ and infinity, respectively), to accommodate the varying object-to-camera distance. Then, as suggested by Gruen and Beyer (2001), images were captured from convergent positions, with large horizontal and vertical bases and included shots obtained rotating the camera $90^{\circ}$ anticlockwise. A total of 12 images were acquired and then uploaded in the Camera Calibrator tool available in PhotoModeler. This tool provides a largely automated solution to detect, identify and measure RAD targets. It also determines a lens distortion model (radial and decentring distortions), principal point offsets, format size and focal length and values describing the calibration quality (overall residual RMSE value, percentage photo coverage, and so on). This calibration workflow was followed with four cameras for the laboratory work but with only one camera for the fieldwork. Four lens distortion parameters were modelled, namely $k_{1}, k_{2}, p_{1}$ and $p_{2}$. For the laboratory experimentation a 'poor' 
calibration was included, obtained by applying an arbitrary change of $\pm 20 \%$ to $k_{1}$ to each of the four camera models (Wackrow and Chandler, 2008). For the fieldwork tests only, a full calibration option was employed.

TABLE II. Differences between the fieldwork and laboratory workflows to process and register point clouds.

\begin{tabular}{lll}
\hline Workflow Step & Fieldwork & Laboratory \\
\hline $\begin{array}{l}\text { (1) Images processed } \\
\text { in PhotoModeler }\end{array}$ & From 4 to 10 images & Only 4 images \\
$\begin{array}{l}\text { (2) Type of camera } \\
\text { and calibration used }\end{array}$ & $\begin{array}{l}\text { One Nikon D80 } \\
\text { Full calibration }\end{array}$ & $\begin{array}{l}\text { Four Nikon D80s } \\
\text { Full and poor calibration }\end{array}$ \\
procedure & GCPs & Only GCPs \\
\hline
\end{tabular}

The default parameters were used for the MVS procedure (Table III) with the exception of 'Texture strength' (default: 0.7), 'Downsample level' (default: 1) and 'Point Spacing' (default: 2). The point cloud alignment process was achieved using different approaches. In the laboratory experimentation, stable and clearly identifiable photogrammetric targets (Fig. 1) were used to ensure a robust co-registration between clouds of different epochs. For the fieldwork, only the point cloud from the first survey was scaled and oriented using targets. This initial survey was used as the 'reference' model to register successive reconstructions. This was achieved by estimating a $3 \mathrm{D}$ similarity transformation that involved manual selection of well-distributed correspondences between the reference model and the 'new' point cloud. Normal practice is to identify natural and/or anthropological features considered stable over the monitoring period (Bakker and Lane, 2017). The open-source software CloudCompare (Girardeau-Montaut, 2019) was used for several aspects in this study. The iterative closest point (ICP) algorithm, implemented in CloudCompare, was also used to optimise the registration between point clouds where significant change is not expected (for example point clouds from the same epoch). A theoretical overlap of $95 \%$ was selected along with the 'adjust scale' option. The minimum root mean square (RMS) improvement between two consequent iterations (indicated as the RMS difference) was $1.0 \mathrm{e}^{-5}$ (default setting). The same settings were maintained for all ICP implementations in this study. The quality of the fitting was determined by calculating the RMS error (RMSE).

For each technique, the point clouds generated were cropped in order to compare the same area, avoiding modelling errors due to a different reconstruction at the periphery of the clouds. However, missing-point zones due to occlusion remained, affecting the result of the comparison. A solution involved adopting a 'closest point set' approach (Besl and McKay, 1992) which selects, for each point of a first cloud, its closest point in a second cloud. This approach allows the removal of points with no correspondence in both clouds, such as occlusion zones.

Post-processed dense point clouds were then compared in a pairwise manner. This method allows production of area-based deformation measurements that provide information on geomorphic change. A pairwise method was also used to estimate the accuracy of a point cloud with respect to a reference point cloud (for example, data obtained with TLS) and to analyse point clouds generated with different approaches (variable number of images; full/poor calibration). The area-based 3D analysis was obtained using tools 
available in CloudCompare, which has been widely used (Micheletti et al., 2015; Nouwakpo et al., 2016). Distances were calculated with a direct cloud-to-cloud comparison performing a closest neighbour interpolation ( $\mathrm{C} 2 \mathrm{C}$ tool in CloudCompare). In addition, a 'multiscale model-to-model cloud comparison' (M3C2 plugin in CloudCompare), which is more suited to rough complex topographies, was employed for the analysis of data collected at Lyme Regis. For both distance-calculation tools, parameters were set based on suggestions found in Lague et al. (2013). Both procedures (C2C and M3C2) allow the production of graphical depictions of change together with the estimation of some metrics about the illustrated deviations in the form of mean error and standard deviation of error, as recommended by Li (1988).

TABLE III. Parameters used for the MVS algorithms implemented in PhotoModeler to generate dense point clouds from the photo-sets collected with the ground-based approach.

\begin{tabular}{|lccc|}
\hline $\begin{array}{c}\text { Minimum no. of } \\
\text { visible images } \\
\text { Minimum angle }\end{array}$ & 3 & Maximum group size & 20 \\
Texture strength & 10 & Window radius & 3 \\
Downsample level & 0.5 & Number of iterations & 2 \\
Point spacing & 0 & Maximise overlap & $\square$ \\
& 1 & Curvature factor & 0.5 \\
& & Increase the window size each & iteration \\
\end{tabular}

\section{RESULTS}

\section{Sensitivity of SfM4C Monitoring}

The histograms in Fig. 3 provide a quantitative assessment of the results for the four scenarios described in section Experimental Environment. Using a full calibration option with a fixed and variable camera configuration, and comparing epoch1 with epoch2 (Figs. 3(a) and (c)), the results are statistically similar achieving, in both cases, a standard deviation $\sigma$ of $0.33 \mathrm{~mm}$ and a mean distance $\mu$ of $0.01 \mathrm{~mm}$. Comparing clouds obtained with a poorly defined lens model with the two camera configurations exhibited large differences: $\sigma=1.82 \mathrm{~mm}$ and $\mu=-2.74 \mathrm{~mm}$ for the variable configuration (Fig. 3(b)); and $\sigma=0.8 \mathrm{~mm}$ and $\mu=-0.02 \mathrm{~mm}$ for the fixed cameras (Fig. 3(d)). A further investigation was conducted after introducing a small change to the slope (epoch3 - Figs. 3(e) to (h)). Analyses considering the four scenarios were conducted comparing epoch1 and epoch3. Statistics calculated using the point cloud generated with a poor calibration achieved the values of $\sigma=2.03 \mathrm{~mm}$ and $\mu=-2.72 \mathrm{~mm}$ for the variable configuration (Fig. 3(f)). However, with fixed cameras these differences dropped to just $\sigma=1.00 \mathrm{~mm}$ and $\mu=-$ $0.005 \mathrm{~mm}$ (Fig. 3(h)) indicating significant monitoring potential, even with a poor camera calibration. 
PARENTE et al. Optimising the quality of an SfM-MVS slope monitoring system using stationary fixed cameras

FULL CALIBATION

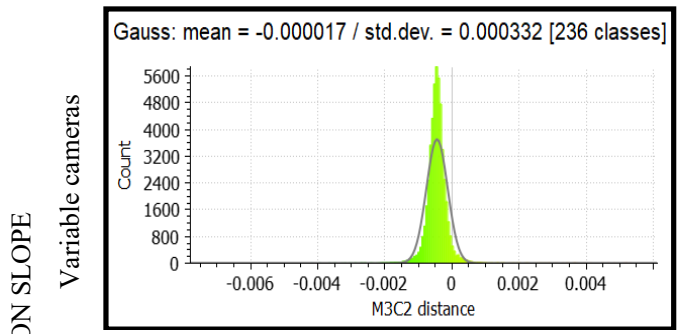

(a)

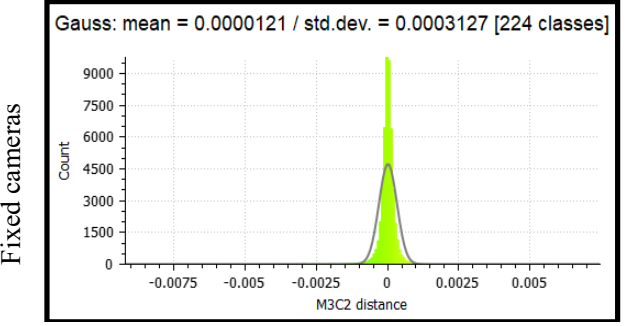

(c)

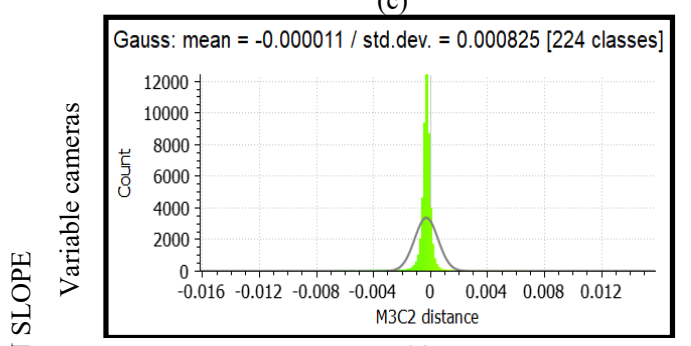

(e)

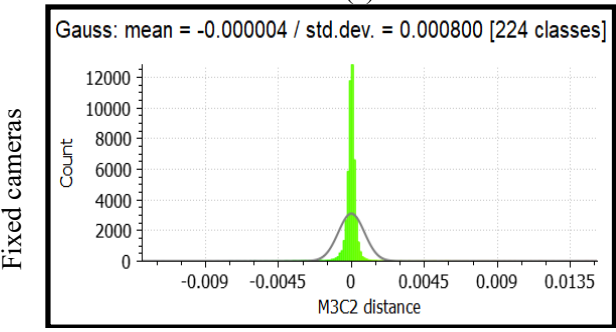

(g)

\section{POOR CALIBRATION}

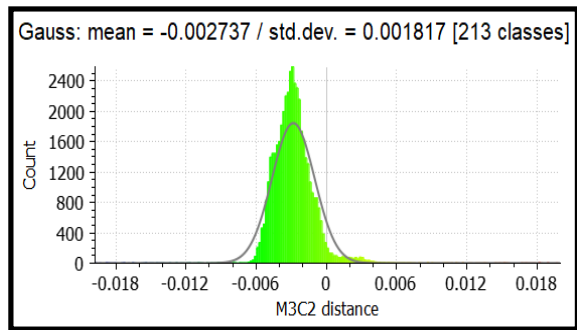

(b)

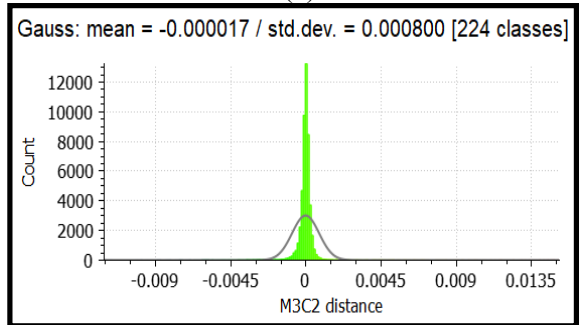

(d)

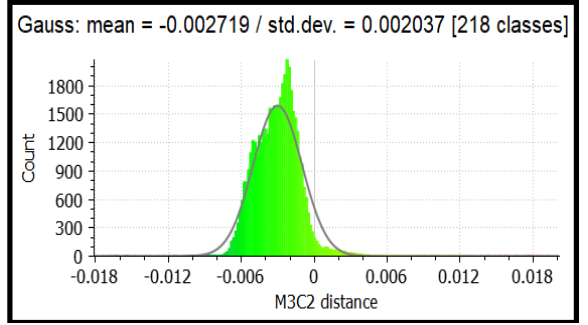

(f)

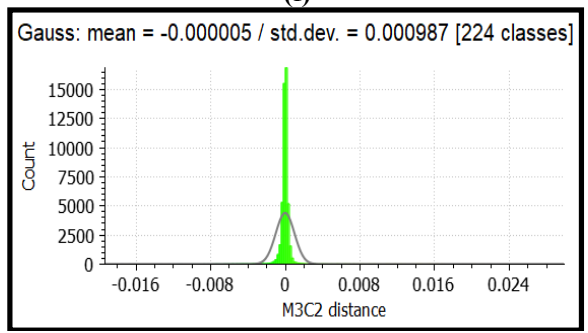

(h)

FIG. 3. Gaussian distribution of distances between point clouds calculated with the M3C2 tool in CloudCompare. The eight histograms ((a) to (h)) shown differences in terms of mean distance and standard deviation values with fixed and variable camera locations for change (epoch3) and no-change (epoch1 and epoch2) scenarios, with both full and poor camera calibration.

Fig. 4 depicts the cloud differences (histograms shown in Figs. 3(e) to (h)) obtained with the $\mathrm{C} 2 \mathrm{C}$ tool in CloudCompare, illustrating these trends graphically. The cloud-to cloud difference obtained by comparing epoch1 and epoch3 associated with a variable 
configuration and poor calibration (Fig. 4(b)) shows many 'false' changes (red) in the study area. This is in contrast to the change/no change representation when a full calibration option was used (Fig. 4(a)), where only real changes were detected. In this case a clear separation between the stable area in blue and the three red zones of excavation are easily identified. Similarly, the ability to distinguish between stable areas and real changes on the slope is evident when cameras are kept in a fixed and mildly convergent configuration, even with poor calibration (Fig. 4(d)). Although additional areas exhibiting highly systematic changes are still visible, they are statistically insignificant when examining the normal distribution curves depicted in Fig. 3(h).

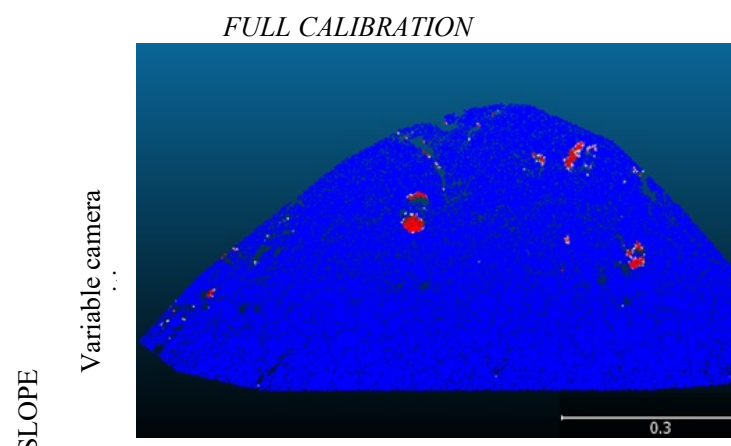

(a)

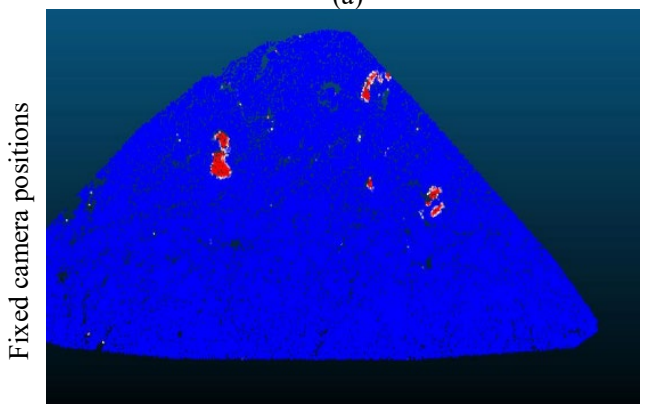

(c)

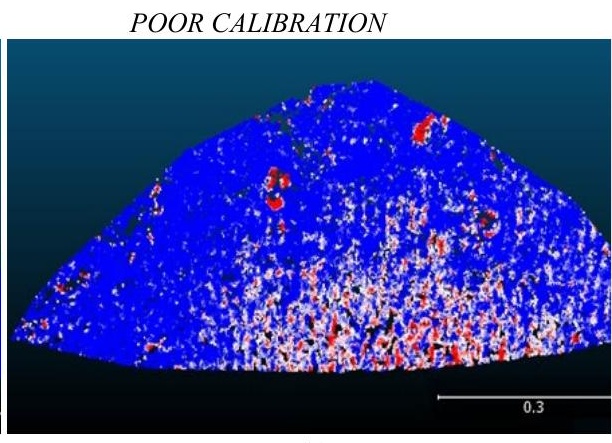

(b)

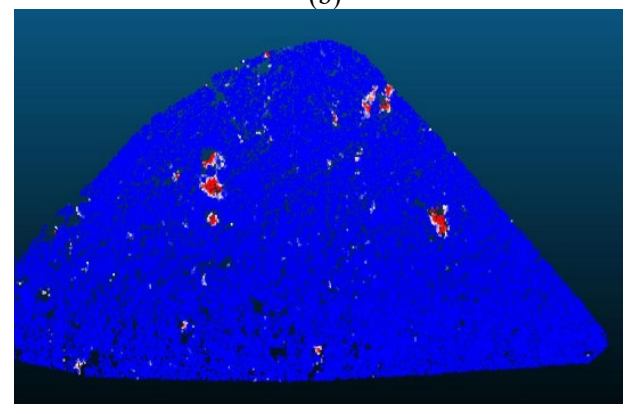

(d)

FIG. 4. C2C comparison between Epoch1 and Epoch3 (change on the slope) using different camera configurations and calibration options. Changes in red; unchanged areas in blue. (a) Variable camera locations full calibration. (b) Variable camera locations - poor calibration. (c) Fixed camera locations - full calibration. (d) Fixed camera locations - poor calibration. Real changes alone in (a), (c) and (d); many false changes in (b).

\section{Terrestrial SfMnC}

This section describes the results obtained from the field test. The term ' $\mathrm{SfM} n \mathrm{C}$ ' is used to indicate an SfM workflow where $n$ images have been processed.

Table IV summarises the results obtained by comparing a four-camera SfM solution with both the TLS and drone-based photogrammetry (DP). Logistically, obtaining a point cloud of the cliff with the TLS approach proved to be the least convenient option, due to the cumbersome equipment, when compared to ground-based imagery and the small drone used for this study. Once it was set up, the laser scanner captured a point cloud of the study area with about 2.1 million points. Table $\mathrm{V}$ shows that on the same day, the SfM4C 
produced a point cloud with approximately 3.5 million points. The SfM4C approach proved to be a much quicker solution in terms of data collection and processing (Table V). Imagery captured from the drone (29 images) generated the least-dense point cloud (about 180 thousand points). Such a result is influenced by the use of the default values for the 'Downsample level' and 'Point spacing' settings of the MVS algorithm. The latter settings were coarser than those used for the SfM4C approach shown in Table III.

The RMSE, mean distance and standard deviation values shown in Table IV highlight a high degree of consistency in co-registering the SfM4C model to those obtained with DP and TLS. This approach was carried out by manually picking four equivalent points in the cloud pairs (centre of targets). In this way, an average 3D error of about $0.01 \mathrm{~m}$ was estimated. When an automatic (ICP algorithm) approach was used in combination with the manual one, the 3D error ranges between $0.05 \mathrm{~m}$ and $0.064 \mathrm{~m}$ (RMSE). Using the $\mathrm{C} 2 \mathrm{C}$ tool to compare the SfM4C cloud with the TLS and drone models, the mean distances ranged between $0.008 \mathrm{~m}$ and $0.088 \mathrm{~m}$, and the standard deviations between $0.005 \mathrm{~m}$ and $0.065 \mathrm{~m}$ respectively. When the 'closest point' tool was adopted, the influences of vegetation and shadow areas were filtered out, as shown in Fig. 5. The SfM4C and TLS models showed a higher uniformity when the two models were filtered and registered through ICP (mean distance of $0.008 \mathrm{~m}$ and standard deviation of $0.005 \mathrm{~m}$ ). Following the same approach, the comparison between SfM4C and DP produced a mean distance and standard deviation of $0.017 \mathrm{~m}$ and $0.012 \mathrm{~m}$ respectively.

TABLE IV. Summary of registration error (RMSE), mean distance and standard deviation values when comparing clouds (C2C) from the SfM4C approach with clouds obtained with the two benchmark techniques (TLS and drone photogrammetry). n/a: not available.

\begin{tabular}{|c|c|c|c|c|c|}
\hline $\begin{array}{c}\text { Clouds } \\
\text { compared }\end{array}$ & Registration & $\begin{array}{c}\text { Closest } \\
\text { point } \\
\text { set }\end{array}$ & $\begin{array}{l}R M S E \\
\text { (m) }\end{array}$ & $\begin{array}{c}\text { Mean } \\
\text { distance } \\
(m)\end{array}$ & $\begin{array}{c}\text { Standard } \\
\text { deviation } \\
(m)\end{array}$ \\
\hline $\begin{array}{l}\text { SfM4C - } \\
\text { TLS }\end{array}$ & Point-pair & No & 0.012 & 0.017 & 0.03 \\
\hline $\begin{array}{c}\text { SfM4C - } \\
\text { TLS } \\
\end{array}$ & Point-pair & Yes & $\mathrm{n} / \mathrm{a}$ & 0.009 & 0.006 \\
\hline $\begin{array}{c}\text { SfM4C - } \\
\text { TLS } \\
\end{array}$ & ICP & No & 0.063 & 0.015 & 0.03 \\
\hline $\begin{array}{c}\text { SfM4C - } \\
\text { TLS }\end{array}$ & ICP & Yes & 0.05 & 0.008 & 0.005 \\
\hline $\begin{array}{l}\text { SfM4C - } \\
\text { drone }\end{array}$ & Point-pair & No & 0.011 & 0.088 & 0.065 \\
\hline $\begin{array}{l}\text { SfM4C - } \\
\text { drone }\end{array}$ & Point-pair & Yes & $\mathrm{n} / \mathrm{a}$ & 0.027 & 0.021 \\
\hline $\begin{array}{l}\text { SfM4C - } \\
\text { drone }\end{array}$ & ICP & No & 0.064 & 0.024 & 0.04 \\
\hline $\begin{array}{l}\text { SfM4C - } \\
\text { drone }\end{array}$ & ICP & Yes & 0.058 & 0.017 & 0.012 \\
\hline
\end{tabular}

In February 2018 a larger photogrammetric dataset was captured. Four point clouds indicated as SfM4C, SfM6C, SfM8C and SfM10C were derived through processing four, six, eight and ten images respectively. Each point cloud differed in terms of point density and processing time (Table V). For the purposes of comparison and validation, the point cloud obtained with TLS in August 2017 was used as the reference cloud and compared through the M3C2 algorithm with the $\mathrm{SfM} n \mathrm{C}$ clouds from February 2018. The results of 
such comparisons are shown in Table VI. The comparison between the reference cloud and the SfM10C cloud produced a mean value of $-0.01 \mathrm{~m}$ and a standard deviation of $0.088 \mathrm{~m}$. When the reference was compared with the SfM4C cloud, the mean value was $-0.007 \mathrm{~m}$ and the standard deviation was $0.095 \mathrm{~m}$. The comparison between point clouds SfM10C and SfM4C obtained on the same day (February 2018), produced low differences (a mean distance of $-0.003 \mathrm{~m}$ and standard deviation of $0.033 \mathrm{~m}$ ).

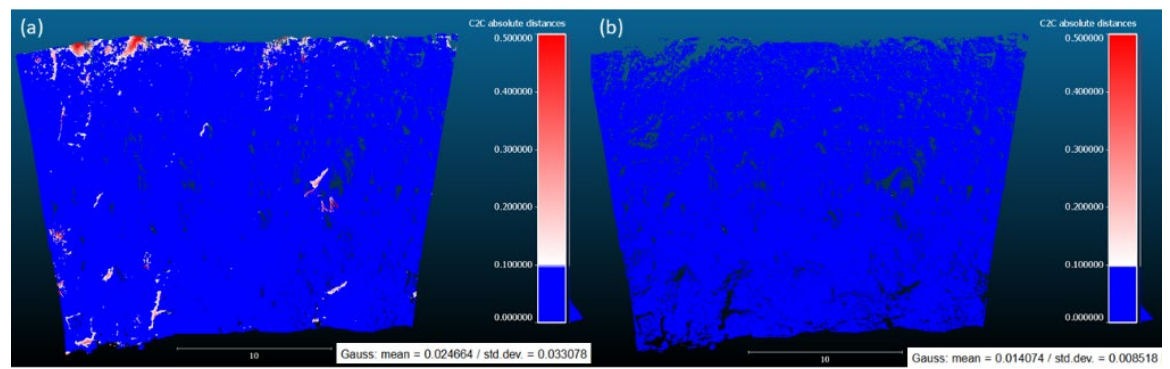

FIG. 5. Example of cloud-to-cloud difference before (a) and after (b) applying the 'closest point set' algorithm (Lyme Regis sea-cliff test site).

TABLE V. Summary of time taken (including data acquisition and processing time) and final point cloud sizes for

TLS, drone photogrammetry and SfM-MVS. *After filteringlcropping the point cloud to the same area of interest. $\mathrm{n} / \mathrm{a}$ : not available.

\begin{tabular}{cccccc}
\hline Date & Technique & $\begin{array}{c}\text { Scans/ } \\
\text { Images }\end{array}$ & $\begin{array}{c}\text { Data collection }+ \\
\text { processing time }\end{array}$ & $\begin{array}{c}\text { Approximate } \\
\text { point count* } \\
\text { (millions) }\end{array}$ & $\begin{array}{c}\text { Average point } \\
\text { density* } \\
\left(\text { pts } / \mathrm{m}^{2}\right)\end{array}$ \\
\hline $22 / 08 / 2017$ & TLS & 1 & $40 \mathrm{~min}+\mathrm{n} / \mathrm{a}$ & 2.1 & 5469 \\
\hline $22 / 08 / 2017$ & $\begin{array}{c}\text { SfM from } \\
\text { drone }\end{array}$ & 29 & $15 \mathrm{~min}+22 \mathrm{~min}$ & 0.2 & 521 \\
\hline $22 / 08 / 2017$ & $\begin{array}{c}\text { Terrestrial } \\
\text { SfM }\end{array}$ & 4 & $4 \mathrm{~min}+44 \mathrm{~min}$ & 3.5 & 9115 \\
\hline $17 / 02 / 2018$ & $\begin{array}{c}\text { Terrestrial } \\
\text { SfM }\end{array}$ & 4 & $4 \mathrm{~min}+57 \mathrm{~min}$ & 5.2 & 13542 \\
\hline $17 / 02 / 2018$ & $\begin{array}{c}\text { Terrestrial } \\
\text { SfM }\end{array}$ & 6 & $6 \mathrm{~min}+1 \mathrm{~h} 44 \mathrm{~m}$ & 6.2 & 16146 \\
\hline $17 / 02 / 2018$ & $\begin{array}{c}\text { Terrestrial } \\
\text { SfM }\end{array}$ & 8 & $8 \mathrm{~min}+2 \mathrm{~h} 30 \mathrm{~min}$ & 6.8 & 17708 \\
\hline $17 / 02 / 2018$ & $\begin{array}{c}\text { Terrestrial } \\
\text { SfM }\end{array}$ & 10 & $10 \mathrm{~min}+2 \mathrm{~h} 58 \mathrm{~min}$ & 7.3 & 19010 \\
\hline
\end{tabular}

TABLE VI. Summary of registration error (RMSE), mean distance and standard deviation values when comparing clouds (M3C2) from the SfM $n$ C approach with clouds obtained with TLS. 8/17 = August 2017; 2/18= February 2018.

\begin{tabular}{|c|c|c|c|c|}
\hline Clouds compared & Registration & $\begin{array}{c}R M S E \\
\quad(m)\end{array}$ & $\begin{array}{c}\text { M3C2 } \\
\text { mean } \\
\text { distance } \\
(m)\end{array}$ & $\begin{array}{c}M 3 C 2 \\
\text { standard } \\
\text { deviation } \\
(m)\end{array}$ \\
\hline TLS (8/17) - SfM10C (2/18) & Manual (5points) & 0.018 & -0.010 & 0.088 \\
\hline
\end{tabular}


PARENTE et al. Optimising the quality of an SfM-MVS slope monitoring system using stationary fixed cameras

\begin{tabular}{lclll} 
TLS (8/17) - SfM8C (2/18) & Manual (5points) & 0.023 & -0.013 & 0.089 \\
\hline TLS (8/17) - SfM6C (2/18) & Manual (5points) & 0.016 & -0.016 & 0.090 \\
\hline TLS (8/17) - SfM4C (2/18) & Manual (5points) & 0.025 & -0.007 & 0.095 \\
\hline SfM4C (2/18) - SfM10C (2/18) & ICP & 0.072 & -0.003 & 0.033 \\
\hline
\end{tabular}

\section{Sea-cliff Change Detection}

In order to further assess the SfM4C approach for monitoring purposes, point clouds of the sea cliff generated using images from the first and the second photogrammetric surveys (22nd and 23rd August 2017) were compared through the CloudCompare M3C2 tool (multiscale model-to-model cloud comparison). The generated point clouds are characterised by a similar point density of about 9000 points $/ \mathrm{m}^{2}$. Registration between the two clouds was achieved with a manual pre-alignment (five point-pair registration) and an automatic refinement (ICP algorithm) approach, respectively produced the following values in terms of RMSE: $0.016 \mathrm{~m}$ and $0.059 \mathrm{~m}$. A cloud-to-cloud difference, describing the distances between points of the two datasets, was used to identify areas of change (Fig. $6(\mathrm{a}))$.

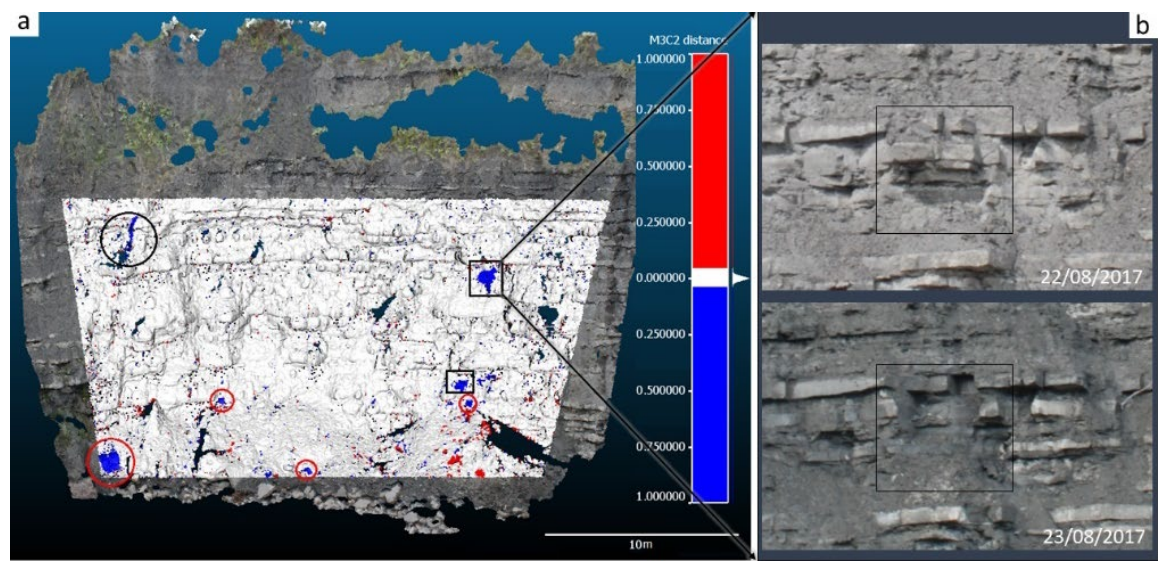

FIG. 6. (a) Colour map (red $>$ white $>$ blue) of the M3C2-based distances computed between the SfM4C surveys of 22nd and 23rd August 2017. Changes detected includes removal of targets (red circles), vegetation (black circle) and rockfall (black rectangles). (b) The two images captured on 22nd and 23rd August 2017 show the material loss detected by the SfM4C monitoring approach.

The comparison revealed changes attributed to three factors: user artefacts; misconstruction of parts of the point cloud; and natural change. The first corresponds to change introduced by the user such as removal of targets from the study area. As a consequence of this operation, the footprint of the operator walking on soft material at the base of the cliff are distinguishable. The second change is due to the presence of vegetation, mainly roots situated in the upper part of the area investigated. While vegetation is static in images from 22nd August, the presence of wind during the second survey affected the point cloud reconstruction. Vegetation appearing in slightly varying positions in the imagery are not reconstructed and this is recorded in the cloud-to-cloud difference with a negative value and interpreted as material loss (blue in Fig. 6(a)). Real changes are linked to collapses from 
the cliff face and relocation of sediment deposited at the cliff toe by waves. The most significant collapse observed on the cliff face occupied an area of about $0.5 \mathrm{~m} \times 1 \mathrm{~m}$, with a depth ranging from 5 to $30 \mathrm{~cm}$. Verification of this collapse was supported by visual inspection of the related photographs before and after the event (Fig. 6(b)).

\section{DISCUSSION}

\section{Fixed Cameras for Improved Monitoring}

While it is recognised that stationary DSLR cameras have been used for monitoring purposes in the past with both stereo (James and Robson, 2014; Roncella et al., 2014) and multistation approaches (Eltner et al., 2017), the effects of poorly calibrated cameras were not investigated. The use of any non-metric camera for spatial measurement using photogrammetry introduces systematic errors affecting the measured image coordinates (Fryer et al., 2007). These errors are mathematically modelled through calibration procedures carried out for each camera. Ensuring that the internal camera geometry and the estimated correction terms remain valid over time is always challenging, particularly when DSLR cameras are used for monitoring on a long-term basis. In such situations, repeating camera calibration procedures is advisable to exclude the influences of internal camera geometrical inaccuracy (Habib et al., 2014). The approach described in this paper demonstrates the capability to compensate for such errors without the need to regularly recalibrate the cameras. Laboratory tests demonstrate the role that camera position can have on the quality of 3D change detection. Results described in Figs. 3 and 4 clearly show improved performance of 3D monitoring when a fixed acquisition configuration is adopted. The impact of adopting a variable camera-location approach when cameras are poorly calibrated is particularly clear in these two figures. If a fully calibrated camera system is adopted, differences between monitoring with a fixed and a variable camera position is indeed minimal (Figs. 3(a), (c), (e) and (g), plus Figs. 4(a) and (c)). With fixed positions, any systematic error introduced by poor camera calibration is minimised (Figs. 3(d), 3(h) and 4(d)).

The tests demonstrate that the hypothesis of a better quality of monitoring with fixed cameras is true as internal camera inaccuracies can be tolerated. It is suggested that, because the imaging angle remains constant and, as a consequence, the same parts of the lens are used to capture the scene at both epochs, this prevents the introduction of errors due to interior camera inaccuracies (or at least cancels their effect). The need to correct deformation errors through calibration is therefore bypassed. The investigated monitoring approach represents an effective alternative to other long-term monitoring methods. Despite this potential, it has to be recognised that measures to fix the camera position is more challenging compared to the controlled environment of the test presented in this paper. Specific solutions should be evaluated to ensure a solid fixture (for example, placing the camera in a sealed and stable box, fixed on a concrete pillar). However, the choice of the right stabilisation measure is dependent on the application (site characteristics, weather conditions and time constraints) and the budget available. A cheaper and logistically friendly solution is being tested by the first author, including the use of poles and a ballhead camera mount, with tensioned cables to increase the stability of the pole. With this approach the camera can be clipped to the mount and removed after image acquisition, 
avoiding issues related to leaving cameras outdoors whilst still ensuring fixed camera positions. Nevertheless, external factors (weather conditions, freeze-thaw cycles and so on) can still affect the stability of cameras (Mallalieu et al., 2017). It is recognised that, in the case of long-term monitoring applications, further research must be conducted to investigate the influences of a continuous deterioration in interior parameters (for example due to temperature variations) and to define the maximum camera movement which can still ensure an accurate measurement. A registration-based approach, to deal with variation in the camera positions, is presented in the section Registration: Limitations and Future Approach.

\section{$\operatorname{SfMnC}$}

Using just four images to monitor surface change with terrestrial SfM photogrammetry proved to be reliable and efficient. The potential to derive a quick and dense 3D reconstruction is critical when continuous near-real-time monitoring is required. The use of an SfM4C approach showed similar capabilities when compared to more established techniques like TLS and DP. In terms of data collection/processing time and information captured (Table V), the SfM4C did allow the generation of a dense point cloud (between 3.5 and 5.2 million points) in less than one hour. The point density achieved is greater than that of the TLS positioned at the same distance from the study site. Considering that each of the three scene reconstruction approaches (TLS, SfM4C and DP) were carried out at the same time, differences between the generated point clouds were primarily due to differences in the scanning resolution of each technique. From such a comparison it is evident that cloud differences are minimal between TLS and SfM4C, with an average change varying between $0.017 \mathrm{~m}$ and $0.008 \mathrm{~m}$ and standard deviation varying between $0.03 \mathrm{~m}$ and $0.005 \mathrm{~m}$ (Table IV). This is in agreement with previous work (Thoeni et al., 2014; Carrivick et al., 2016) where differences between a TLS model and an SfM-MVS cloud are evaluated. However, the SfM-MVS cloud in these studies were obtained by processing about 20 images from smaller camera-to-object distances (between 2.1 and $7.5 \mathrm{~m}$ ). The potential of the SfM4C approach is also supported by values of measurement error and relative error (respectively $0.03 \mathrm{~m}$ and $0.0015 \mathrm{~m}$ without applying the closest point set algorithm), which are similar or even better than those achieved in similar studies reviewed by Eltner et al. (2016).

Cropping clouds obtained with different techniques to a common area improves the quality of the cloud comparison. However, zones with missing points due to occlusions can still influence the comparison. Such influences can be avoided by using the closest point tool available in CloudCompare. This tool filters out noise and areas where points from both clouds are not found. The impact of such a tool is an improvement in the quality assessment of the compared clouds, producing lower values in terms of mean distance and standard deviation (0.008-0.009 $\mathrm{m}$ and $0.005-0.006 \mathrm{~m}$ respectively).

The registration methods used to align the clouds generated with SfM4C and TLS produced dissimilar results in terms of RMSE (Table IV). The point-pair approach and ICP algorithm generated an error of $0.012 \mathrm{~m}$ and $0.063 \mathrm{~m}$ respectively, due to a different approach in computing the RMSE. In the point-pair approach, the RMS value is calculated based only on the point-pairs. When using the ICP tool, the RMS value summarises the mean residuals between a higher point-to-point correspondence, which is set by the operator by adjusting the 'random sampling limit' value (a default value of 50000 was used). Furthermore, because only four points were used, redundancy is low and the RMSE values 
are not representative of the registration accuracy. In this case, mean and standard deviation values are more valuable for registration accuracy assessment. Negligible differences in terms of standard deviation and mean value are estimated when registering the SfM4C cloud with both reference clouds (TLS and drone). Small improvements are achieved in registration accuracy when using an ICP registration approach (Table IV).

Using the SfM-MVS workflow with a larger number of images (6, 8 and 10) has shown little impact on the quality of the model generated and a linear trend is not detectable (Table $\mathrm{V}$ and VI). Comparing SfM4C with SfM10C, the high similarity between these two approaches is demonstrated by a relatively low mean distance $(-0.003 \mathrm{~m})$ and standard deviation $(0.033 \mathrm{~m})$ (Table VI). The latter standard deviation value is in agreement with the values shown in Table IV, when manual and automatic registration is used (no closest point set) to compare SfM4C with TLS. This is again influenced by differences in occlusion areas within the two clouds, which are reduced when using a 10-camera approach. However, the difference between using 4 or 10 images is minimal; both show similar monitoring potential. In fact, comparing both clouds (SfM4C and SfM10C) to the TLS one (Table VI), a larger standard deviation is produced (about $0.09 \mathrm{~m}$ ) as a consequence of changes that occurred on the cliff face in the six months between August 2017 and February 2018.

\section{Registration: Limitations and Future Approach}

Point cloud comparison for change detection between different epochs, or for quality assessment of two scanning techniques, requires the establishment of a common and stable reference system. Depending on the application, a number of approaches are generally adopted to minimise errors in the similarity transformation (rotation, translation and scaling) applied to the point clouds. Strategies employed in this paper included placing photogrammetric targets of known coordinates (Liang et al., 2014) in the study area. Another option is to use camera position coordinates to georeference the point clouds (Turner et al., 2014). However, measuring targets and cameras positions over time can be time consuming and hence costly. The challenge of installing and surveying an appropriate number of targets at each epoch led to alternative solutions being examined. The use of a fixed camera set-up has the theoretical advantage of generating models aligned in the same local reference system. Unfortunately, cameras can never be regarded as perfectly fixed and stable over time. Also, to obtain true and scaled changes, at least one distance between two known features has to be defined at each epoch, although it is recommended to add more control points to increase the redundancy and produce more reliable results.

When cameras are not installed on solid supports, aligning two point clouds by manually identifying the same features in the point clouds and adopting a manual (pointpair tool) and an automatic (ICP algorithm) registration still produces errors. There are a range of challenges, including: (i) ability of the operator to select the same feature for each epoch; (ii) dissimilarity in points distribution on both clouds; and (iii) the time waiting for the ICP processing. Al-Rawabdeh et al. (2016) proposed a robust registration option which relies on the knowledge of the coarse image position at each exposure. When cameras are not fixed and measuring image positions is not feasible, a quick and low-cost solution involves the use of the scale-invariant feature transform (SIFT) (Lowe, 2004) across multiple photographic epochs. To the authors' knowledge, this was first adopted in a comparable context by Feurer and Vinatier (2018), who used the SIFT algorithm to capture 
digital elevation models (DEMs) using a sequence of archival aerial imagery. The same approach can be adopted for terrestrial image sets and eradicates, elegantly, all unstable feature points. Applying SIFT to multitemporal images automatically registers the multiimage epochs into a single referenced image block. Once the first epoch's images are orientated (and optionally scaled), all point clouds generated will maintain a common spatial reference system. Assuming that a sufficient number of features in the study area remain unchanged over time and are correctly matched, the proposed 'registration SIFT' approach can be adopted for both the variable and fixed cameras configurations, eliminating both the need for laborious registration procedures (for example Liang et al., 2014; Turner et al., 2014) or adopting strategies for camera movement corrections (for example Roncella et al., 2014).

\section{CONCLUSION}

This research has investigated the use of multiple yet fixed DSLR cameras and SfMMVS photogrammetry for monitoring purposes. Two study sites have been used to investigate the potential, including a close-range laboratory environment and a sea cliff. A TLS survey and a drone-based photogrammetric survey were used to provide comparative data. The results demonstrate an improvement in monitoring quality when a fixed-camera approach is adopted. Such an approach improves the measurement accuracy of the SfMMVS workflow, even when the lens model is poorly determined. Also, outputs of this study suggest that a traditional SfM-MVS workflow, where tens to hundreds of images are captured, can be replaced by a four-image (SfM4C) approach. Not only will this reduce field time and data processing but it will still return an accurate result. The use of fixed cameras helps to ensure that accurate differences can be determined, even if camera calibration is sub-optimal. This investigation identified further research questions and reveals potential for developing an automated near-real-time monitoring system that exploits a convergent fixed-camera configuration and an automatic registration method through exploiting the basic SIFT algorithm.

\section{ACKNOWLEDGEMENTS}

This research was presented at the 3rd Virtual Geoscience Conference thanks to the support of a travel bursary awarded to the first author by the Remote Sensing and Photogrammetry Society.

\section{REFERENCES}

Al-Rawabdeh, A., Al-Gurrani, H., Al-Durgham, K., Detchev, I., He, F., El-Sheimy, N. and HabiB, A., 2016. A robust registration algorithm for point clouds from UAV images for change detection. International Archives of Photogrammetry, Remote Sensing and Spatial Information Sciences, 41(B1), 765-772.

BAKKER, M. and LANE, S. N., 2017. Archival photogrammetric analysis of river-floodplain systems using structure from motion (SfM) methods. Earth Surface Process and Landforms, 42(8): 1274-1286.

BESL, P. J. and MCKAY, N. D., 1992. A method for registration of 3-D shapes. IEEE Transactions on Pattern Analysis and Machine Intelligence, 14(2): 239-256.

Brown, D. C., 1971. Close-range camera calibration. Photogrammetric Engineering, 37(8): 855-866.

CARrivick, J. L., SMith, M. W. and QuincEY, D. J., 2016. Structure from Motion in the Geosciences. Wiley, New York, USA. 197 pages.

DOUSKOS, V., KALISPERAKIS, I. and KARRAS, G., 2007. Automatic calibration of digital cameras using planar chess- 
board patterns. 8th Conference on Optical 3-D Measurement Techniques, Zurich, Switzerland. (1): 132-140.

Eltner, A., Kaiser, A., CAstillo, C., Rock, G., Neugirg, F. and Abellán, A., 2016. Image-based surface reconstruction in geomorphometry - merits, limits and developments. Earth Surface Dynamics, 4: 359-389.

Eltner, A., Kaiser, A., Abellan, A. and Schindewolf, M., 2017. Time lapse structure from motion photogrammetry for continuous geomorphic monitoring. Earth Surface Processes and Landforms, 42(14): 2240-2253.

FEURER, D. and VinATIER., F., 2018. Joining multi-epoch archival aerial images in a single SfM block allows 3-D change detection with almost exclusively image information. ISPRS Journal of Photogrammetry and Remote Sensing, 146: 495 - 506.

Fryer, J., Mitchell, H. and Chandler, J., 2007. Applications of 3D Measurement from Images. Whittles, Caithness, Scotland. 304 pages.

FORT, D. S., MARTIN, P. L., ClARK, A. R. and DAVIS, G. M., 2007. Lyme Regis phase II coast protection and slope stabilisation scheme, Dorset, UK - the influence of climate change on design. In Landslides and Climate Change: Challenges and Solutions (Eds. R. McInnes, J, Jakeways, H. Fairbank and E. Mathie). Proceedings of the International Conference on Landslide and Climate Change, Ventnor, Isle of Wight, UK. 513 pages: 419 428.

Gillan, J. K., Karl, J. W., Elaksher, A. and Duniway, M. C., 2017. Fine-resolution repeat topographic surveying of dryland landscapes using UAS-based structure-from-motion photogrammetry: assessing accuracy and precision against traditional ground-based erosion measurements. Remote Sensing, 9: article 437. 24 pages.

Girardeau-Montaut, D., 2019. CloudCompare (version 2.x; GPL software), EDF R\&D, Telecom ParisTech. http://www. cloudcompare.org/ [Accessed 19th January 2019].

Gruen, A. and BEYER, H. A., 2001. System calibration through self-calibration. Chapter 7 in Calibration and Orientation of Cameras in Computer Vision (Eds. A. Gruen and T. S. Huang). Springer Series in Information Sciences, Vol. 34. Heidelberg and Berlin, Germany. 229 pages: 163-194.

GÜLCH, E., 1984. Geometric calibration of two CCD-cameras used for digital image correlation on the Planicomp C100. International Archives of Photogrammetry and Remote Sensing, 25(A3): 159-168.

HaBIB, A., Detchev, I. and KWAK, E., 2014. Stability analysis for a multi-camera photogrammetric system. Sensors, 14(8): 15084-15112.

HABIB, A. and Morgan, M., 2005. Stability analysis and geometric calibration of off-the-shelf digital cameras. Photogrammetric Engineering \& Remote Sensing, 71(6): 733-741.

HEIKKILÄ, J., 2000. Using circular control points. IEEE Transactions on Pattern Analysis and Machine Intelligence, 22(10): 1066-1077.

Irvine-Fynn, T. D. L., SAnZ-Ablanedo, E., Rutter, N., Smith, M. W. and Chandler, J. H., 2014. Measuring glacier surface roughness using plot-scale, close-range digital photogrammetry. Journal of Glaciology, 60(223): 957-969.

JAMES, M. R. and RoBSON, S., 2014. Sequential digital elevation models of active lava flows from ground-based stereo time-lapse imagery. ISPRS Journal of Photogrammetry and Remote Sensing, 97: 160-170.

KenefiCK, J. F., GYER, M. S. and HARP, B. F., 1972. Analytical self-calibration, Photogrammetric Engineering \& Remote Sensing, 38(11): 1117-1126.

Lague, D., Brodu, N., Leroux, J., Rennes, G., Rennes, U. and De Beaulieu, C., 2013. Accurate 3D comparison of complex topography with terrestrial laser scanner: application to the Rangitikei canyon ( N-Z ), ISPRS Journal of Photogrammetry and Remote Sensing, 82: 10-26.

LI, Z., 1988. On the measure of digital terrain model accuracy. Photogrammetric Record 12(72): 873-877.

LIANG, Y.-B., ZHAN, Q.-M., CHE, E.-Z., CHEN, M.-W. and ZHANG, D.-L., 2014. Automatic registration of terrestrial laser scanning data using precisely located artificial planar targets. IEEE Geoscience and Remote Sensing Letters, 11(1), 69-73.

Lowe D. G. 2004. Distinctive image features from scale-invariant keypoints. International Journal of Computer Vision, 60(2): 91-110.

Mallalieu, J., Carrivick, J. L., Quincey, D. J., Smith, M. W. and James, W. H. M., 2017. An integrated structure-from-motion and time-lapse technique for quantifying ice-margin dynamics. Journal of Glaciology, 63(242): 937-949.

MASON, S., RÜTHER, H. and SMIT, J., 1997. Investigation of the Kodak DCS460 digital camera for small-area mapping. ISPRS Journal of Photogrammetry and Remote Sensing, 52(5): 202-214.

MAY, V. J., 2003. Golden Cap to Lyme Regis. Chapter 4 in Coastal Geomorphology of Great Britain (Eds. V. J. May and J. R. Hansom). Geological Conservation Review Series, No. 28. Joint Nature Conservation Committee, Peterborough, UK. 754 pages: 4/1- 4/9. https://pdfs.semanticscholar.org/1dd0/909c3fb148d6b 03ed928f71dba15718ce5b0.pdf [Accessed: 20th August 2017].

Micheletti, N., ChandleR, J. H. and LANE, S.N., 2015. Investigating the geomorphological potential of freely 
PARENTE et al. Optimising the quality of an SfM-MVS slope monitoring system using stationary fixed cameras

available and accessible structure-from-motion photogrammetry using a smartphone. Earth Surface Processes and Landforms, 40(4): 473-486.

NOUWAKPO, S. K., WeLTZ, M. A. and MCGWIRE, K., 2016. Assessing the performance of structure-from-motion photogrammetry and terrestrial LiDAR for reconstructing soil surface microtopography of naturally vegetated plots. Earth Surface Processes and Landforms, 41(3): 308-322.

Piermattei, L., CARTURAN, L., GUARNieri, A., 2015. Use of terrestrial photogrammetry based on structure-frommotion for mass balance estimation of a small glacier in the Italian alps. Earth Surface Processes and Landforms, 40(13): 1791-1802.

REMONDINO, F. and Fraser, C., 2006. Digital camera calibration methods: considerations and comparisons. International Archives of Photogrammetry, Remote Sensing and Spatial Information Sciences, 36(5), 266-272.

Roncella, R., Forlani, G., ForNARI, M. and DiOTRI, F., 2014. Landslide monitoring by fixed-base terrestrial stereo-photogrammetry. International Annals of Photogrammetry, Remote Sensing and Spatial Information Sciences, 2(5): 297-304

Rieke-Zapp, D., Tecklennburg, W., Peipe, J., Hastedt, H. and Haig, C., 2009. Evaluation of the geometric stability and the accuracy potential of digital cameras - comparing mechanical stabilisation versus parameterisation. ISPRS Journal of Photogrammetry and Remote Sensing, 64(3): 248-258.

SanZ-Ablanedo, E., RodrígueZ-PÉrez, J. R., Armesto, J., Flor, M. and TaboadA, Á., 2010. Geometric stability and lens decentering in compact digital cameras. Sensors, 10(3): 1553-1572.

Seitz, S. M., CuRless, B., Diebel, J., Scharstein, D. and Szeliski, R., 2001. A comparison and evaluation of multi-view stereo reconstruction algorithms. IEEE Computer Society Conference on Computer Vision and Pattern Recognition, New York, USA. 1: 519-528.

SnAVely, N., SeitZ, S. M. and SzEliski, R., 2006. Photo tourism: exploring photo collections in 3D. ACM Transactions on Graphics, 25(3): 835-846.

Thoeni, K., Giacomini, A., Murtagh, R., Kniest, E., Modelling, M., Comparison, C. and Modelling, T., 2014. A comparison of multi-view 3D reconstruction of a rock wall using several cameras and a laser scanner. International Archives of Photogrammetry, Remote Sensing and Spatial Information Sciences, 40(5): 23-25.

TURNER, D., LuCIEER, A. and WALLACE, L., 2014. Direct georeferencing of ultrahigh-resolution UAV imagery. IEEE Transactions on Geoscience and Remote Sensing, 52(5): 2738-2745.

UlLman, S., 1979. The interpretation of structure from motion. Proceedings of the Royal Society of London, Series B 203(1153): 405-426.

WACKROW, R. and CHANDLER, J. H., 2008. A convergent image configuration for DEM extraction that minimises the systematic effects caused by an inaccurate lens model. Photogrammetric Record, 23(121): 6-18. 